\title{
Sédimentation, volcanisme et présence humaine dans le bassin de Venosa (Basilicata, Italie) au Pléistocene moyen : exemple du site de Notarchirico.
}

\author{
David LEFEVRE (1), Jean-Paul RAYNAL (2), Gérard VERNET (2), \\ Thierry PILLEYRE (3), Marcello PIPERNO(4), Serge SANZELLE (3), \\ Jean FAIN (3), Didier MIALLIER (3), Michèle MONTRET (3).
}

(1) Laboratoire de Géomorphologie et d'Etude du Quaternaire, UPR 7559 CNRS - ERA 37 CRA Université des Sciences et Technologies de LILLE, F59655 VILLENEUVE D'ASCQ cedex.

(2) Institut du Quaternaire, Université de BORDEAUX I, UMR 9933 CNRS Avenue des Facultés, F33405 TALENCE cedex.

(3) Laboratoire de Physique Corpusculaire, IN2P3 - CNRS, Université Blaise Pascal, F63177 AUBIERE Cedex.

(4) Dipartimento di Discipline Storiche, Università di Napoli, Federico II, NAPOLI.

Résumé : La Formation de Piano Regio et la Formation de Tufarelle composent le remplissage du Bassin de Venosa. La sédimentation y est contrôlée par l'activité volcanique du Monte Vulture dont il a enregistré les différentes phases d'activité au cours de la fin du Pléistocène ancien et du Pléistocène moyen. A Notarchirico, plusieurs épisodes volcaniques représentés par des ponces, des retombées fines et grossières et leurs différents produits de remaniement ont interrompu la dynamique sédimentaire lacustre et entraîné des modifications environnementales bien particulières. Les occupations successives du site suggèrent une fréquentation opportuniste du milieu volcanique par les Hominidés du Pléistocène moyen.

Mots-clés : Volcanisme, Pléistocène moyen, Paléolithique ancien, Hominidés.

Abstract : Direct tecto-volcanism is the main process involved in the sedimentary filling of the Venosa lacustrine Basin. The former interpretations had insisted on sedimentary process and underestimated distal volcanic facies which can be residuals and make more difficult correlation attempts. The sedimentary series exposed at the Notarchirico Middle Pleistocene prehistoric site is interpreted as the superposition of initially positive grading sequences which failed because of a general trend to negative grading deposition due to the volcanic activity. Several volcanic episodes represented by pumice falls and coarse and fine ash-falls and following epiclastic processes have interrupted the lacustrine sedimentation and induced specific environmental transformations. The sequences are separated by stone pavements which represent polygenic surfaces considered as stratigraphic hiatus. Hominids were present immediatly after every volcanic event and this can be considered as an opportunist exploitation of volcanic environment.

Key-words : Volcanism, Middle Pleistocene, Lower Palaeolithic, Hominids.

Les gisements de la région de Venosa (Basilicata) représentent une des séquences les plus longues et les mieux documentées du Paléolithique inférieur en Italie.

Dès le XIXè siècle, la richesse paléontologique et préhistorique du bassin de Venosa est reconnue à Terranera (Nicolucci, 1877). Les dépôts fluviolacustres du bassin donnent alors lieu aux premières interprétations stratigraphiques (Guiscardi, 1880) en liaison avec les études conduites sur 
l'appareil volcanique du Monte Vulture (De Lorenzo, 1899, 1904). Un siècle plus tard, ils font l'objet de travaux détaillés (Piccaretta et Ricchetti, 1970) et sont intégrés dans une analyse géomorphologique régionale (Neboit, 1975).

Des fouilles sont entreprises dès le début du siècle dans la localité de Terranera (Rellini, 1915 ; Mochi, 1913, 1916) puis, à la suite à de nouvelles prospections (Topa, 1932), à Loreto (Rellini, 1932, D'Erasmo, 1932). La localité de Loreto va faire l'objet de fouilles systématiques (Blanc, 1953 ; Chiappella,1966 ; Barral et al, 1978, Barral et Simone, 1982, 1984), d'études géologiques (Segre,1978 ; Baïssas, 1980) et paléontologiques (Bonifay, 1977 ; Angelleli et al., 1978 ; Durante et Settepassi, 1978 ; Caloi, Palombo, 1979).

Plus récemment, les recherches se sont portées sur le site voisin de Notarchirico (Segre et al., 1982, 1984 ; Piperno, 1987, 1989, 1990 ; Belli et al., 1991). Un programme d'études chronostratigraphiques est conduit sur ce site et dans le bassin depuis 1990. Nous en présentons ici les premiers résultats en insistant sur le contrôle volcanique de la sédimentation, de la paléogéographie, voire des occupations préhistoriques.

\section{1 - LE BASSIN DE VENOSA.}

La région de Venosa correspond à un bas plateau situé entre Appennino et Murge. Du point de vue structural (Boenzi et al., 1987), elle appartient à la fosse bradanique, bassin d'âge Néogène, comblé par une séquence sédimentaire régressive : faciès argileux de plateforme, passant à des faciès sableux coquilliers littoraux (sables fauves) puis à des faciès conglomératiques polygéniques de bordure ayant livré des restes d'Archidiscodon meridionalis (Segre, 1978) (figure 1).

La surface de la dépression, développée aux dépens des conglomérats, est à une altitude de 400/420 m au Piano de Cammera et s'incline doucement en direction du Nord-Est.

Une dépression allongée, orientée Ouest-Nord-Ouest - Est-Sud-Est, large de 2 à $4 \mathrm{~km}$, est encaissée d'une cinquantaine de mètres sous la surface du plateau. Elle est interprétée comme la paléo-vallée d'un organisme fluviatile s'écoulant vers le Sud-Et en direction du Bradano et dont la géométrie traduit l'importance du contrôle tectonique (Piccarreta et Richetti, 1970 ; Neboit, 1975). Son remplissage est constitué de faciès correspondant à des environnements sédimentaires fluviatiles, palustres/lacustres, associés à des lahars et des téphras, plus ou moins remaniées, contemporaines de l'activité du Vulture (Piccarreta et Richetti, op. cit.). Nous avons nommé cette unité morpho-lithostratigraphique Formation de Tufarelle (Lefèvre et al., 1991) (figure 2). 
A la base, plusieurs séquences de conglomérats et de sables à larges stratifications obliques incurvées emboitées correspondent à un environnement sédimentaire de barres graveleuses dans des rivières à chenaux anastomosés. En montant dans la série, la fraction sableuse s'enrichit en minéraux volcaniques et les séquences sableuses terminales présentent une alternance de lits sombres à pyroxènes et de lits clairs à ponces ; des bancs lenticulaires de tufs ponceux terminent la deuxième séquence sableuse. La dernière séquence correspond à un système de type lahar/épi-lahar, qui met en place un conglomérat fortement hétérométrique à blocs de lave claire pris dans une matrice de sables grossiers à stratifications entrecroisées. Ces conglomérats qui se retrouvent à des dizaines de kilomètres du volcan en direction du SudEst, indiquent un drainage vers le Bradano. Verticalement, dans la partie axiale de la paléo-vallée, la série se différencie : aux faciès épiclastiques grossiers, succède une alternance de bancs de calcaires correspondant à des environnements sédimentaires lacustres et/ou palustres, de bancs de tephra plus ou moins remaniées et de retombées directes de scories trachytiques. En montant, la série devient de moins en moins épiclastique et de plus en plus lacustre : bancs de calcaires lacustres plus épais, surfaces d'émersion durcies et encroutées, bancs de limons jaunes tachetés, paléosols hydromorphes. La succesion observée sur le site préhistorique de Loreto est typique de la Formation de Tufarelle (figure 3). Rappelons que la limite Brunhes-Matuyama a été localisée par Baïssas (1980) dans le niveau 37. L'ensemble de la série serait donc à rapporter à la fin du Pléistocène inférieur et à la première partie du Pléistocène moyen.

En position plus latérale, les faciès diffèrent : paléosols (sols rouges, sols bruns) moulant des paléotopographies de vallons, co-ignimbrites altérées (caractères andiques), conglomérats disposés soit en pavages de versants, soit en lentilles d'ordre métrique correspondant à des écoulements dans des chenaux de courts affluents issus des collines voisines, épiclastites. Dans la partie inférieure de la série, importantes accumulations de tufs ponceux, avec plusieurs retombées directes bien individualisées au sein desquelles s'observent des variations chimiques syn-éruptives. Temporai-rement, nous regroupons ces faciès au sein de la Formation de Piano Regio.

Les différents faciès volcaniques identifiés ont pour origine le massif du Monte Vulture, appareil volcanique complexe qui domine le bassin à une vingtaine de $\mathrm{km}$ à l'ouest. Plusieurs phases d'activité y ont été distinguées et datées : les plus anciennes manifestations éruptives sont datées de 860-830 Ka par la méthode du K/Ar ; des épisodes plus récents sont datés de 670/660 $\mathrm{Ka}$ et $430 / 500 \mathrm{Ka}$ (Cortini, 1975). Des résultats récents confirment plus ou moins ces résultats et attestent de plusieurs phases d'activité volcanique au cours du Pléistocène moyen, jusqu'à la limite des stades isotopiques 6 et 5 (Villa, 1991; La Volpe et Principe, 1990; Bonadonna et al., 1993; Laurenzi et al., 1993). 
Mise à part peut-être la base de son remplissage, la série sédimentaire du bassin de Venosa est contemporaine de cette activité. Les études en cours dans le bassin devraient permettre d'établir une téphrostratigraphie détaillée et de la corréler avec les diverses phases identifiées sur le Monte Vulture.

En résumé, l'histoire du bassin peut être provisoirement résumée comme suit. A partir d'une surface de remblaiement construite vers la fin du Pliocène, s'installe et s'encaisse un réseau hydrographique qui va modeler au cours du Pléistocène inférieur une large paléo-vallée, encaissée une centaine de mètres en contre-bas. A la charnière du Pléistocène inférieur et du Pléistocène moyen, les premières manifestations volcaniques désorganisent les écoulements fluviatiles en apportant un volume important de matériaux que les cours d'eau sont incapables d'évacuer. La paléovallée évolue en dépression parcourue par des écoulements de moindre énergie, plus localisés, issus probablement des versants et remaniant du matériel volcanique et/ou occupée par des lacs peu profonds ou des marécages, alors que se poursuit l'activité volcanique : progressivement, la dépression va se combler de 30 à $50 \mathrm{~m}$ de sédiments. Une importante activité néotectonique (failles, bombements) va orienter la mise en place d'un nouveau réseau hydrographique (Fiumara de Venosa) s'écoulant maintenant vers le Nord-Ouest, en direction de l'Ofanto, et induire une incision généralisée des formations plus anciennes, au cours du Pléistocène supérieur, mettant ainsi en terrasse les formations du Pléistocène moyen.

\section{2 - LA SERIE DE NOTARCHIRICO.}

Plusieurs niveaux archéologiques ont été dégagés sur le site préhistorique de Notarchirico. Les outillages appartiennent à l'Acheuléen moyen italien. La faune est dominée par l'Elephant antique, les cervidés et les bovidés. Un fragment de fémur humain a été découvert en 1985 (Segre et al. , 1982; Piperno and Segre, 1982; Segre and Piperno, 1984; Piperno, 1987, 1990; Belli et al. 1991).

La série sédimentaire de Notarchirico s'est déposée en bordure de bassin, au pied d'un versant entaillant les dépôts conglomératiques pliopléistocènes. Elle représente sans doute un faciès de bordure dont l'âge est sensiblement celui de la partie supérieure de la Formation de Tufarelle (Loreto), voire plus récent. Les essais de datation conduits antérieurement sur ce site indiquent en effet tous un âge Pléistocène moyen.

\section{1 - Lithostratigraphie.}

Cette première approche ne concerne que la partie sommitale de la série, actuellement visible dans le Musée de site, entre le sol $\mathrm{F}$ à la base et la surface des dépôts, les unités antérieures n'étant pas accessibles (figure 4). 
Ces observations complètent les descriptions stratigraphiques antérieures (Belli et al., 1991 ; Lefevre et al., 1991).

De la base au sommet, on distingue :

Unité 3. Unité volcano-sédimentaire basale, à petites stratifications entrecroisées, métriques et peu creusées, soulignées par des scories et des minéraux libres et se terminant par un ensemble de lamines. Les scories sont rares et roulées. Les minéraux libres n'ont pas de verre volcanique adhérant à leur faces et sont plus ou moins émoussés ou en fragments de prismes. Ce sont majoritairement des clinopyroxènes verts et quelques orthopyroxènes. Cette retombée est remaniée et reconcentrée en milieu aqueux (échantillon TL 9002). Un sédiment graveleux surmonte l'ensemble. Puissance $0.50 \mathrm{~m}$.

Paléo-surface F. pavage de galets dont les éléments sont apparemment emballés dans la matrice graveleuse du sommet de l'unité 3.

Unité 2. Complexe volcano-sédimentaire intermédiaire, d'une puissance de 3,25m, composé de :

2.1. Retombée fine grise, dans laquelle on observe une forte concentration de minéraux libres et de rares scories. Les minéraux libres sont des pyroxènes verts en fragments de prismes. Cette retombée a été reconcentrée en milieu aqueux (échantillon TL 9003). Des figures de charge l'affectent et incorporent des ilôts de la tephra blanche 2.2. susjacente.

2.2. Retombée fine blanche, contenant de rares fragments de pyroxènes, du quartz et des fedspaths, à structure en échardes et microbulles. II s'agit d'une retombée acide directe.

\subsection{Retombée fine gris-rosé, avec minéraux libres et scories.}

L'ensemble 2.1 à 2.3 est épais de $0,20 \mathrm{~m}$ et déformé : involutions, encapuchonnements et injections correspondant à des figures de charge. A noter que la retombée 2.3, incorporée aux déformations, présente une partie supérieure non déformée, peu épaisse en coupe frontale et déprimée par endroit.

2.4. Retombée fine blanche micacée, puissance $0,20 \mathrm{~m}$, localement plus épaisse dans de micro-dépressions au toit de 2.3. Les lits sont moins épais vers le haut et séparés par des lamines carbonatées indurées. 
2.5. Sables volcaniques gris clairs à pyroxènes, cimentés par des carbonates, comportant des lentilles et fuseaux centimétriques d'éléments plus grossiers (tephras remaniées). Puissance $0.10 \mathrm{~m}$.

Paléo-surfaces E et E1 : nombreux galets, objets lithiques et ossements dans la partie sommitale des sables de 2.5.

2.6. Complexe volcanosédimentaire d'une puissance moyenne de $1,00 \mathrm{~m}$, avec de bas en haut des limons sableux puis argileux surmontés de sables fins entrecoupés de nombreux apports volcaniques gris fins incluant des ponces fibreuses. Ces dépôts sont parfois reconcentrés dans des rigoles et présentent de nombreuses figures de charge. Dans la partie Ouest du site, le sommet de 2.6 est très riche en ponces.

Paléo-surface D : au sommet de 2.6, dans une matrice plus grossière et plus litée, très riche en minéraux volcaniques mélanocrates.

2.7. Sables volcaniques leucocrates contenant des ponces, à granoclassement vertical positif, conservés uniquement dans la partie Ouest du site. Puissance $0.40 \mathrm{~m}$.

2.8 Sables fins verts à intercalations de lamines argileuses, discordants en biseau sur 2.7. Puissance maximale $0.45 \mathrm{~m}$. La charge fine est de plus en plus importante vers le sommet où on note des lits argileux de plus en plus minces, entrecoupés de carbonates, et des figures de dessication (fissures horizontales et verticales) et de remaniement (boulettes). Passage progressif à 2.9.

2.9 Argile brun-rosé, à structure prismatique, présentant des figures de dessication. Des figures de charge correspondent à l'emplacement des objets de la paléo-surface $\mathrm{C}$.

Paléo-surface C : au toit de 2.9. Puissance $0.30 \mathrm{~m}$.

2.10 Sables hétérométriques à charge volcanique importante. Plusieurs sous-unités comblent une paléo-dépression. Puissance $0.60 \mathrm{~m}$.

Paléo-surface B : lit ondulé de galets jointifs formant un pavage au toit de 2.10.

Unité 1. Complexe volcano-sédimentaire supérieur, composé de :

1.1 Dans le secteur Nord, graviers et sables lités gris-vert à granoclassement vertical (tendance plus argileuse au sommet). Puissance 0.30 
m. Dans le secteur Sud, sables non lités, limono-argileux, contenant des graviers dispersés dans la masse, plus nombreux à la base.

1.2 Argiles brunes plus ou moins sableuses, contenant des galets mous de sapropel et galets. Puissance $0.50 \mathrm{~m}$.

1.3 Complexe ponceux gris-vert. Puissance $0.60 \mathrm{~m}$. 1.31 : ponces fines ; 1.32 : ponces grossières chenalisées ; 1.33 : ponces fines ; 1.34 : ponces grossières ; 1.36 : ponces fines.

\subsection{Graviers et galets lités. Puissance maximale $0.50 \mathrm{~m}$.}

Paléo-surface 7 : objets associés au dernier lit de graviers.

1.5 Dans la partie Est de la zone, plusieurs niveaux de tephras remaniées passant à une sédimentation sableuse fine de plus en plus litée et argileuse, avec une alternance de lits détritiques fins (argileux) et de lits carbonatés. On note la présence de figures en chenaux avec failles de tassement et figures de charges. Puissance $1.25 \mathrm{~m}$.

1.6 Tephras grossières, plus ou moins mélanocrates avec quelques galets ; contact basal par figures de charge. Abondants minéraux volcaniques libres (pyroxènes) et scories de dimensions variées plus ou moins roulées caractérisent des retombées directes remaniées et concentrées en milieu aqueux (échantillon TL 9010). Le sommet est plus régulièrement lité en fuseaux et consolidé. Puissance $0.50 \mathrm{~m}$.

Unité 0 :

0.1 Chenaux colmatés de colluvions fines pédogénisées.

0.0 Horizon labouré.

\section{2 - Interprétation séquentielle.}

Les principaux faciès rencontrés relèvent de dynamiques lacustres, de versant ou volcaniques. Nous nous bornerons ici à proposer une première interprétation dynamique de la série.

L'enregistrement correspond globalement à une (des) séquence(s) d'accumulation sous contrôle volcano-tectonique, en relation avec une remontée continue et/ou saccadée du niveau général du lac suite à un engorgement. On observe un balancement entre une sédimentation lacustre terrigène (fine), voire organogène (sapropel), et une sédimentation détritique plus ou moins grossière. 
Du point de vue de l'analyse séquentielle, la série s'interprète comme une succession de six séquences positives avortées à cause de l'activité volcanique qui introduit systématiquement une dérive séquentielle négative (sauf dans la sous-séquence précédant la paléo-surface $\mathrm{C}$ ). Les 5 premières ont enregistré une déstabilisation des versants conduisant à la constitution de plages lacustres à galets. Nous établissons ici une relation directe entre les épendages téphriques résultant de l'activité éruptive, le déclenchement des apports de versants et la formation subséquente de plages grossières (figure 5).

L'existence de pavages (paléo-surfaces) s'interprète de la façon suivante : des apports détritiques latéraux chargés en éléments grossiers, mis en place par une dynamique de versant, subissent un lavage entraînant une élutriation et la concentration relative des éléments grossiers qui peut aboutir à la disposition jointive en "pavage" (paléo-surfaces B et $\mathrm{F}$ par exemple). Dans ce cas, les pavages marquent selon nous une discontinuité stratigraphique avec les séquences sus-jacentes et représentent donc une surface polygénique à valeur de diasthème dont la signification en terme de durée ne peut être que difficilement évaluée et pose problème lors de l'interprétation archéologique.

\section{3 - Un problème archéologique : "les restes de l'éléphant".}

Les "restes de l'éléphant" appartiennent à la séquence sédimentaire 1 qui repose sur le niveau $B$ de la série stratigraphique de Notarchirico (Cassoli et al., 1991) (figure 6). Le niveau $B$, qui s'étend sur plusieurs centaines de mètres carrés, est un pavage régulier, assemblage jointif de galets, accessoirement d'artéfacts et d'os, formant en coupe un lit unique ("stone-line") à contact planaire avec la couche sous-jacente.

Au dessus, reposent des sables lités, gris-vert, à granoclassement positif, parfois graveleux à la base au contact des galets, plus argileux au sommet. Ils contiennent de nombreux restes osseux entiers, parfois en connexion, principalement de Daim et de bovidés et une rare industrie lithique. Dans la zone Sud, ils passent à des sables limono-argileux contenant des graviers dispersés dans la masse, sans litage apparent.

Dans le "secteur de l'éléphant", le pavage forme une dépression aux contours irréguliers, allongée suivant un axe Nord-Ouest-Sud-Est et profonde d'une quinzaine de centimètres au maximum. Elle est comblée de graviers et sables grossiers, contenant des galets de dimension moyenne. La plus grande partie des restes lithiques et osseux repose sur (cas du crâne et des défenses) ou est contenue dans ce sédiment graveleux (cas de la mandibule, retournée et enfoncée dans le gravier) ; d'autres se trouvent à sa base, au contact ou inclus dans le pavage B. Verticalement, ces graviers passent aux sables lités granoclassés qui, latéralement, reposent directement sur le pavage. 
La validité de l'association faune/outils, en tant qu'assemblage en position primaire, dépend de l'interprétation dynamique de l'enveloppe sédimentaire. clure :

Les caractères stratonomiques et sédimentologiques permettent d'ex-

- un apport par fluage sur un versant (apport en masse) des différents éléments du dépôt (sédiment grossier comprenant les éléments du pavage, restes osseux de l'éléphant, industrie lithique),

- un transport et dépôt par un courant de forte énergie, impliquant plusieurs remaniements et l'association composite d'éléments en position secondaire.

Les évidences de terrain ne permettent pas d'interpréter l'ensemble comme une séquence positive dont la base serait représentée par le pavage $B$ : le contact basal planaire et l'absence de large stratification incurvée de chenal conduisent à rejeter une mise en place par un organisme de type fluviatile de haute énergie.

Nous interprétons le pavage comme la conséquence d'une suite d'événements dynamiques dans un contexte de plage (lacustre lato sensu) : évacuation des fines, tassements différentiels... Son extension exclut catégoriquement une origine anthropique. Sa surface polygénique marque, à notre avis, une discontinuité stratigraphique avec la séquence sableuse sus-jacente et représente donc un plancher (toit/mur) et non le début de cette séquence 1.

Le remplissage graveleux de la dépression peut soit appartenir à cette histoire polygénique, soit représenter la base de la séquence supérieure. Dans ce dernier cas, l'absence de figure de ravinement montre qu'il ne fait que combler une dépression préexistante formée par de légères ondulations du pavage, conséquences probables de la compaction du niveau fin sous-jacent à $B$ et d'une élutriation des fines.

Les caractères (granoclassement, litage) de la séquence sableuse ou gravelo-sableuse 1 conduisent à retenir l'hypothèse d'une mise en place par ruissellements et à rejeter l'hypothèse d'un apport en masse par fluage.

Cependant, dans l'état actuel de la fouille, le rapport entre les restes crâniens de l'éléphant et le sédiment encaissant n'est pas entièrement éclairci :

- ces restes crâniens reposent-ils sur le dallage du niveau B, logés dans le creux de la dépression qui a pu servir à les caler ? La lentille de 
graviers représente alors un bourrage, le crâne servant de piège à sédiment.

- reposent-ils sur la lentille de gravier qui comblait déjà la dépression ? II est certain que lorsque les défenses se sont cassées, le gravier était en place.

Quelle que soit l'hypothèse retenue, les sables lités sont venus ensuite envelopper le crâne (effet de bourrage nettement visible).

En conclusion, ces observations conduisent à distinguer la mise en place des sédiments de celle du crâne (pas d'apport en masse), mais ne permettent pas d'aller plus loin en ce qui concerne le mode de dépôt de ce dernier (flotté puis échoué, ou mort sur place), à plus forte raison donc les causes de sa mort (naturelle ou résultant d'un piégeage ou d'une chasse). Reste posée la question de la réalité de l'association éléphant/restes lithiques. Si association il y a, tous les objets présents dans la lentille de gravier lui appartiennent-ils ? Sinon, comment les distinguer? ceux enchâssés dans le dallage semblent en tout état de cause à écarter.

\section{3 - DISCUSSION.}

Ces premières études montrent très clairement que le volcanisme contrôle étroitement la sédimentation dans le bassin de Venosa. Les interprétations antérieures ont sans aucun doute sous-estimé les nombreux faciès volcaniques distaux et les processus épiclastiques qui forment l'essentiel des séquences des formations reconnues (Tufarelle et Piano Regio).

A Notarchirico, plusieurs épisodes volcaniques représentés par des ponces, des retombées fines et grossières et leurs différents produits de remaniement ont interrompu la dynamique sédimentaire lacustre et entraîné des modifications environnementales bien particulières.

Dans ce milieu soumis à des modifications brutales, I'Homme était présent. Cette présence opportuniste - adaptation au milieu volcanique - était peut être motivée par la quête de nourriture carnée plus facile à obtenir massivement immédiatement après chaque éruption : altération du couvert végétal et concentration animale aux points d'eau, présence de marécages, morts subites d'animaux et en particulier un grand nombre d'oiseaux, affaiblissement des proies par blessures, piégeages naturels dans des coulées boueuses, inhalations de gaz carbonique et anhydride sulfureux, blessures du palais des herbivores liées au fluor des aérosols... phénomènes dont l'ampleur a pu être mesurée sur des exemples historiques et contemporains et qui ont déjà été évoqués à plusieurs reprises à propos des peuplements préhistoriques du 
Massif Central français (Daugas et Raynal, 1991 a et b ; Raynal et Daugas, 1984, 1989, 1991 ; Raynal et Sanzelle, 1989).

Du strict point de vue chronologique, les dates proposées pour le site de Notarchirico ne contredisent pas les données radio-chronologiques obtenues par d'autres méthodes sur le site (Belli et al., 1991 ; Piperno et al., 1990) (figure 7) et sont compatibles avec ce que l'on connaît de l'âge de l'activité du Monte Vulture dont les ultimes phases éruptives datent de la fin du Pléistocène moyen (Cf supra). Elles demandent à être vérifiées (par de nouvelles mesures), controlées (par d'autres méthodes) et confrontées aux données obtenues sur l'appareil du Monte Vulture. Les micrommamifères récoltés au sommet de 2.5 indiquent également un âge Pléistocène moyen (SALA, 1991).

Ces différents aspects constitueront l'essentiel des travaux des années à venir. II est en effet particulièrement important - du strict point de vue des industries préhistoriques - d'établir clairement l'âge des séries de Venosa, en raison des implications quand à l'ancienneté du peuplement européen et de l'organisation interne des complexes "acheuléens" d'Italie. II est non moins important de préciser les modalités d'adaptation aux paléoenvironnements volcaniques et les activités quotidiennes de ces premiers européens.

Remerciements : les auteurs remercient le Professeur Marcello Piperno (Universita di Napoli) pour son accueil et son aide lors des missions de terrain à Venosa, le Professeur Serge Occhietti (UQAM, Montréal) pour sa lecture critique du manuscrit, la Soprintendenza Speciale al Museo Nazionale Preistorico L. Pigorini di Roma, les Universités de Bordeaux 1, Clermont II et Lille 1, le CNRS et I'IN2P3 pour leur soutien au projet. 


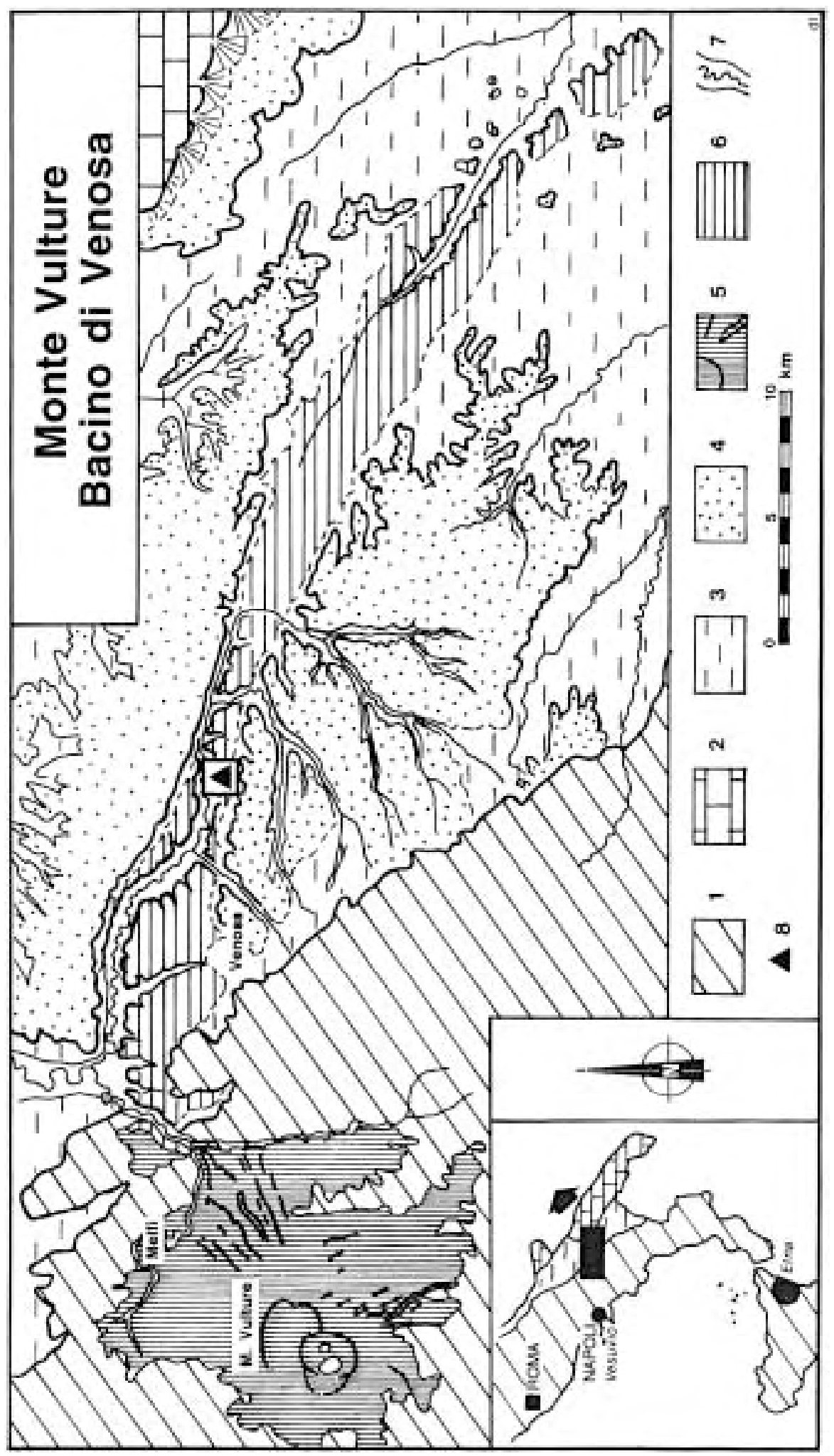

Figure 1 : Carte morphostructurale de la région de Venosa - Monte Vulture. 1 : Apennin méridional.

2 : Plateau calcaire des Murge (plate-forme carbonatée de l'avant-pays Apulien). 3 : Fosse Bradanique. Argiles bleues de Gravina puis Sables de Monte Marano. Pliocène. 4 : Fosse Bradanique. Conglomérats d'Irsina. Pliocène supérieur / Pléistocène inférieur. 5 : Complexe volcanique du Monte Vulture. Pléistocène moyen.

6 : Dépôts volcano-sédimentaires et fluvio-lacustres de Venosa. Pléistocène moyen. 7 : Dépôts alluviaux de fonds de vallées. 8 : Localisation du secteur étudié. 

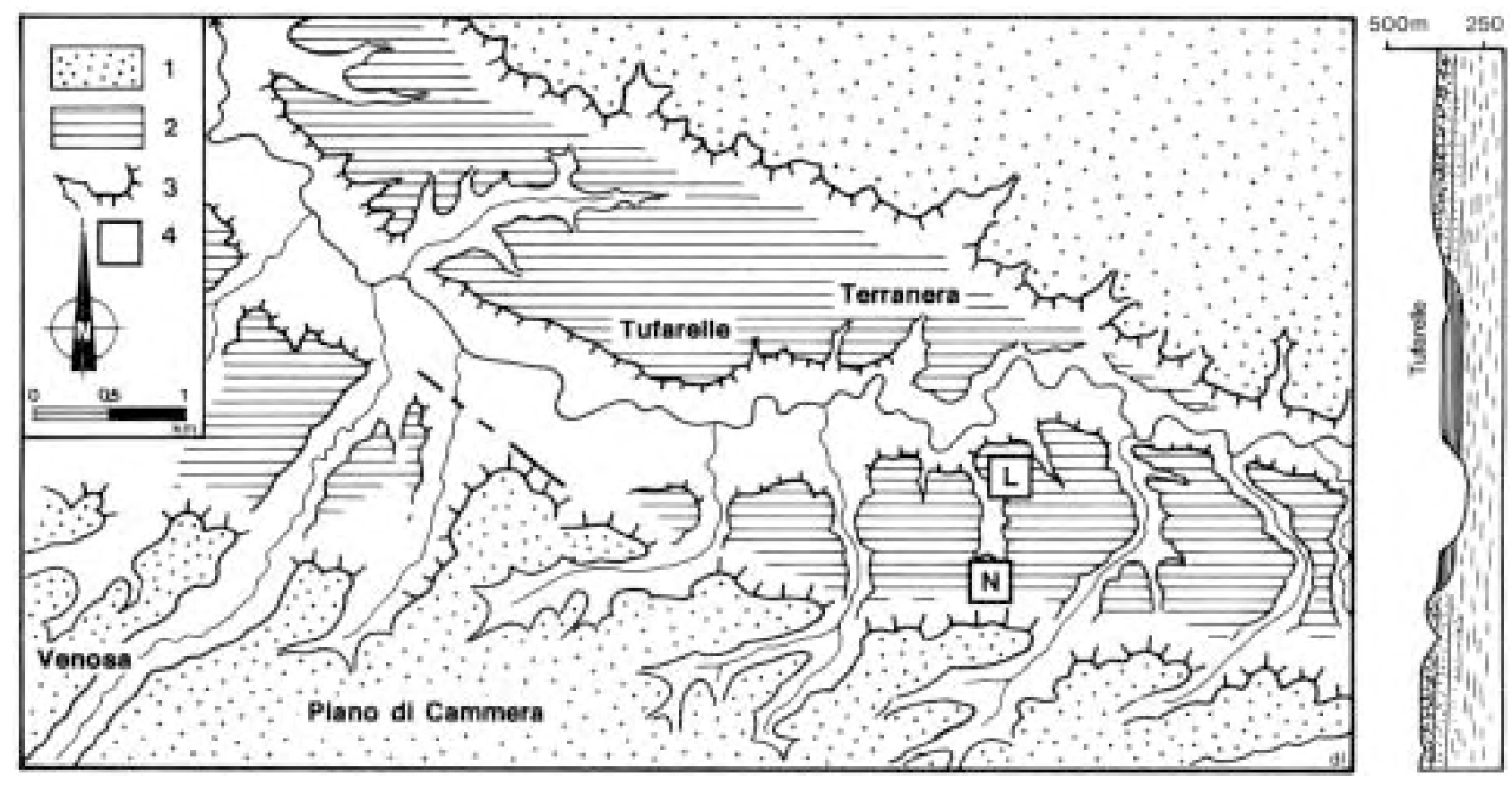

Figure 2 : Localisation des principaux gisements étudiés et localités de la région de Venosa. 1 : Conglomérat plio-pléistocène d'Irsina.

2 : Dépôts volcano-sédimentaires et fluvio-lacustres de Venosa. 3 : Rebord de terrasse.

: Sites préhistoriques et paléontologiques de Notarchirico $(\mathrm{N})$ et Loreto $(\mathrm{L})$. 


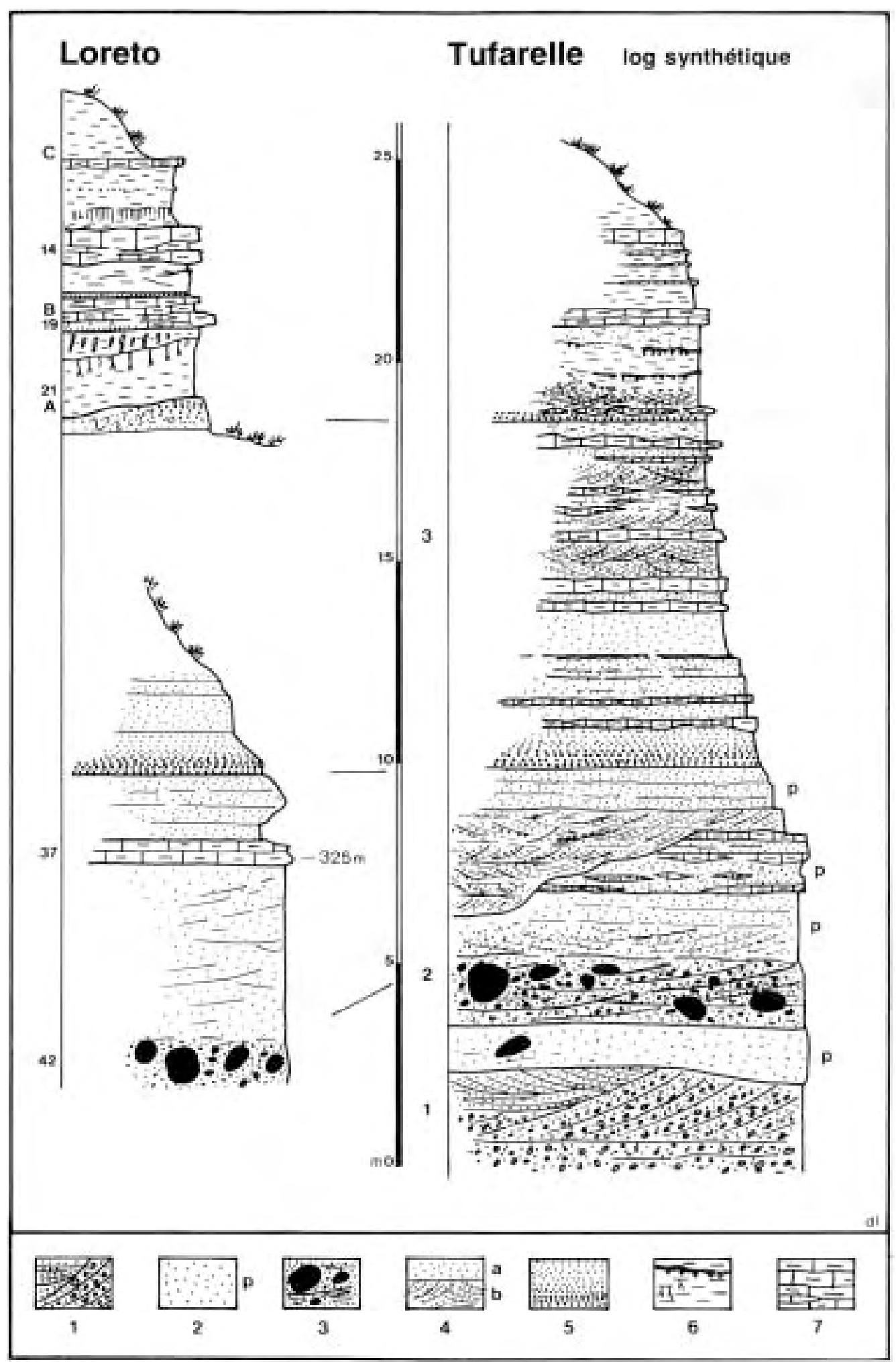

Figure 3 : Log synthétique de la formation de Tufarelle et de la localité de Loreto. 1 : Dépôt fluviatile grossier. 2 : Ponces.

3 : Lahar et épi-lahars.

4 : Tephra et épiclastites.

5 : Retombée directe.

6 : Silts et bancs durcis carbonatés avec réseau de racines.

7 : banc calcaire. 
Lefevre et al.,Bull. S.P.F., 1994, tome 91, $n^{\circ} 2$, 103-112, manuscrit - 15 -

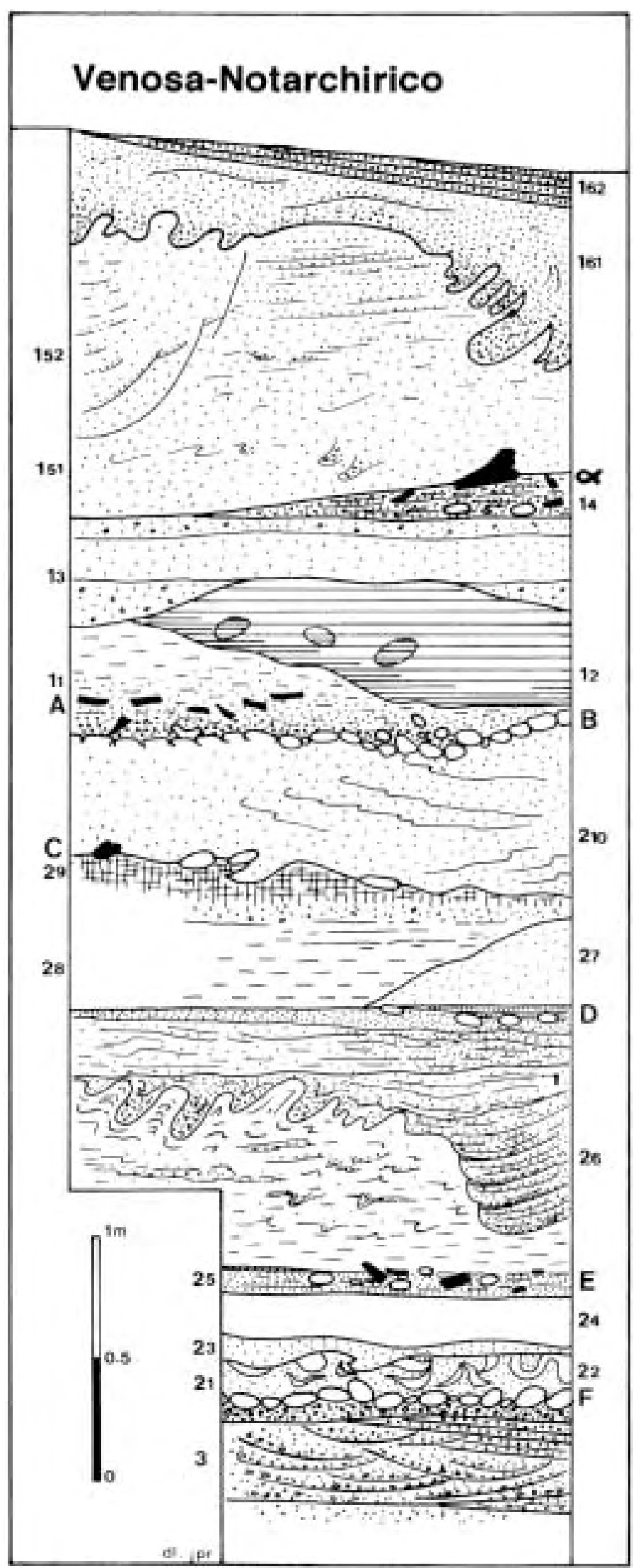

Figure 4 : Log synthétique de la série de Venosa-Notarchirico. Légende dans le texte. 

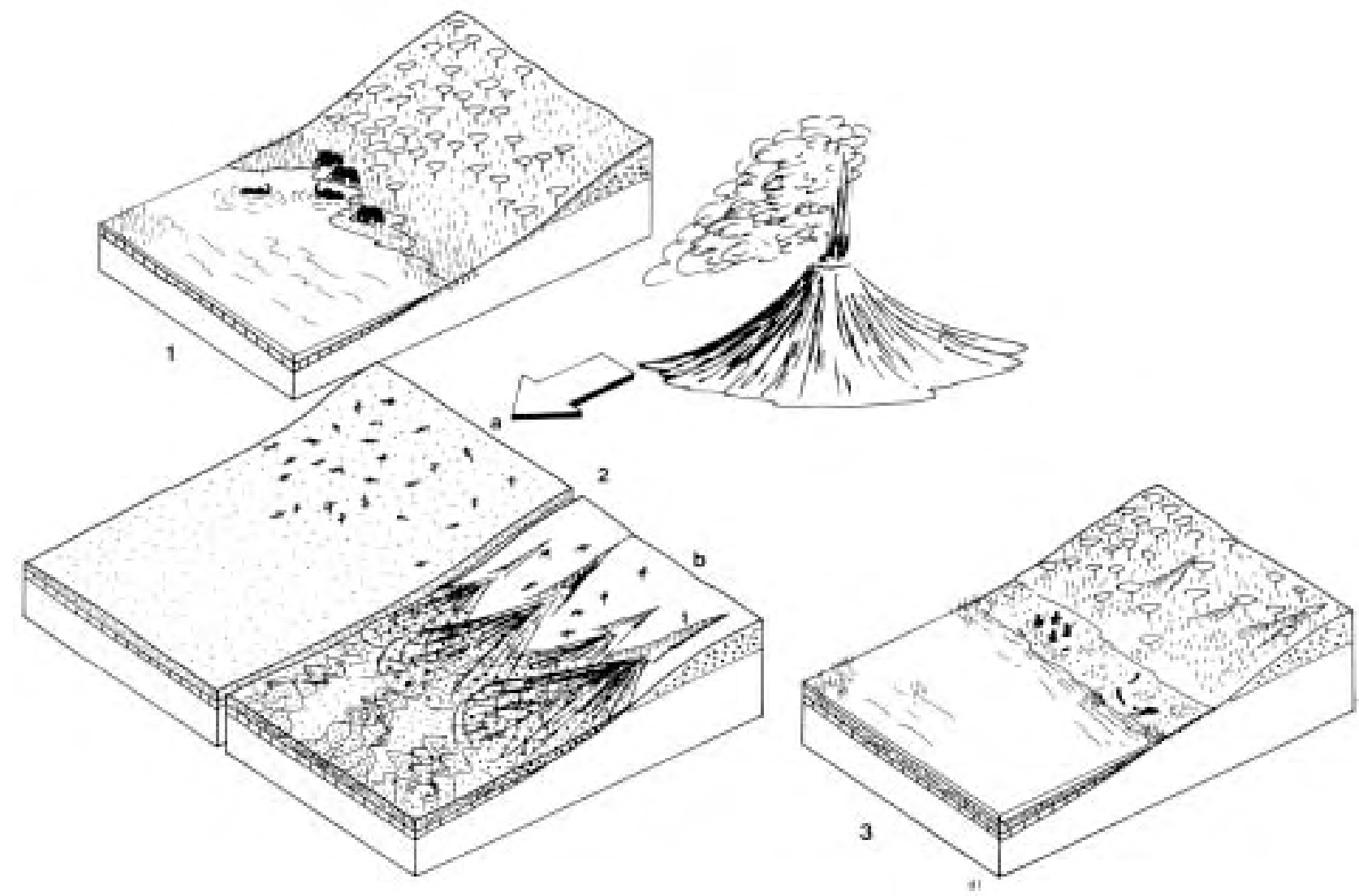

Figure 5 : Dynamique d'une séquence type sous contrôle volcanique. 1 : Episode lacustre/palustre. Equilibre des versants.

2 : Eruption volcanique.

a : accumulation de tephra

b : remaniements de tephra, glissements sur les versants.

3 : Episode lacustre : équilibre sur les versants, formation de plage lacustre par lavage des accumulés en pied de versant. 


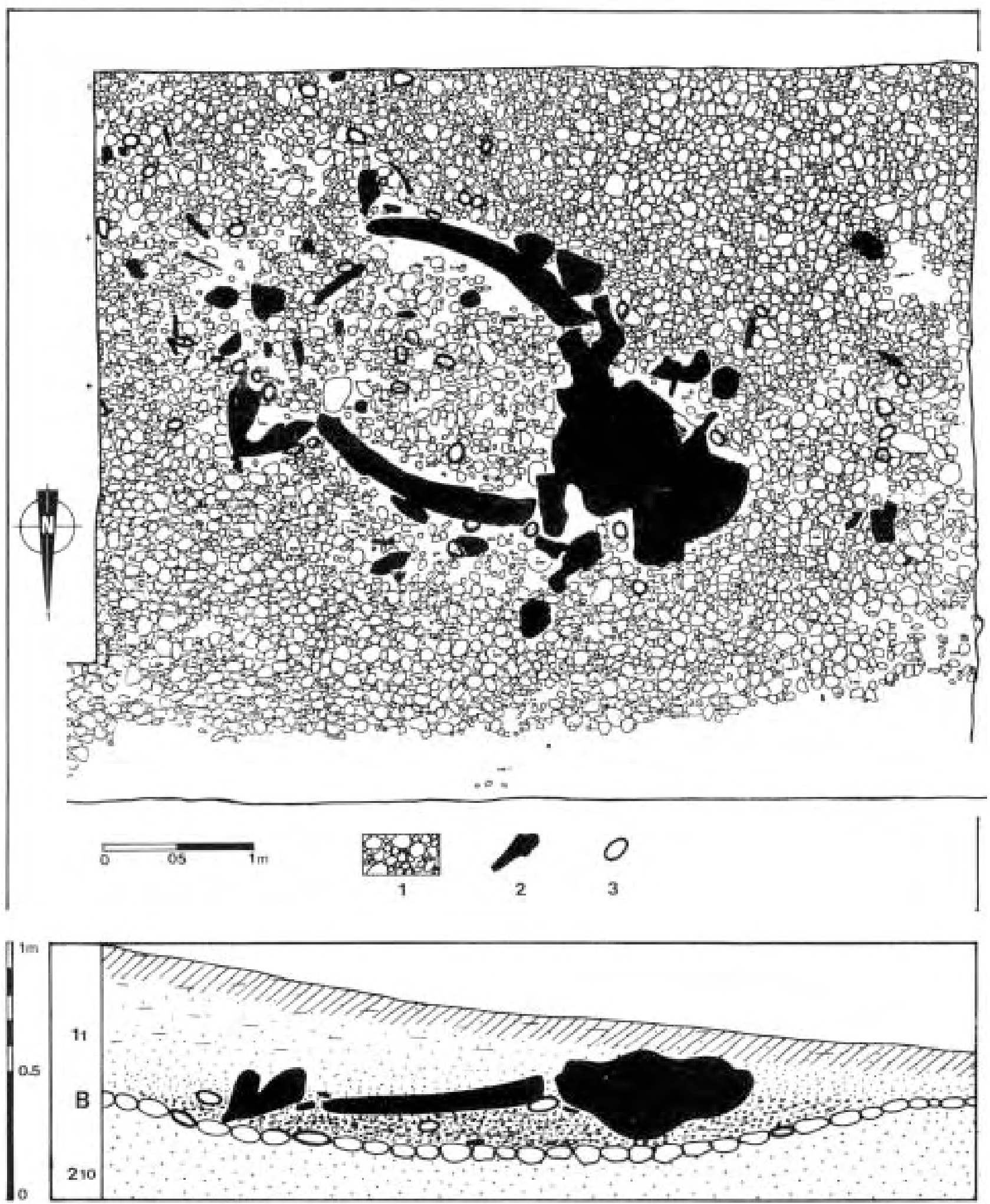

Figure 6 : Venosa-Notarchirico, secteur de l'Elephant. Plan et coupe synthétique.

1 : Paléo-surface B (relevé par photogrammétrie).

2 : Restes de faune.

3 : Artéfacts (d'après Cassoli et al., 1991). 
Lefevre et al.,Bull. S.P.F., 1994, tome 91, $n^{\circ} 2,103-112$, manuscrit - 18 -

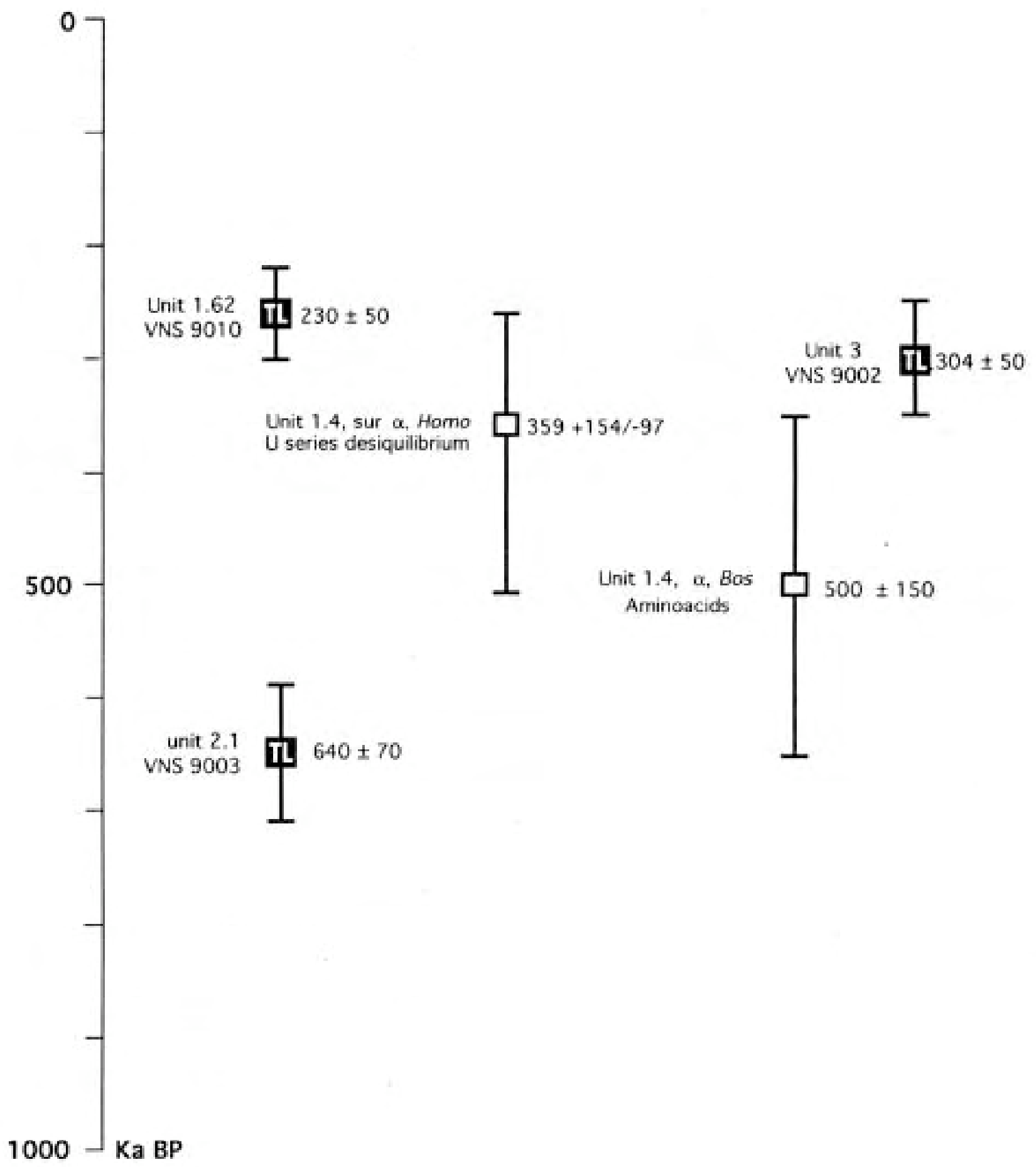

Figure 7 : Datations obtenues sur la séquence de Venosa-Notarchirico. 


\section{Références}

Angelelli F., Caloi L., Malatesta A., Palombo M.R. (1978) - Fauna quaternaria di Venosa: cenni preliminari. Atti XX Riun. Sc. Ist. It. Preist. Protost., Firenze, pp. 133-140.

Baïssas P. (1980) - Données paléomagnétiques et sédimentologiques sur les dépôts de la coupe de Loreto, Venosa. Bull. Mus. Anthrop. Préhist. Monaco, 24, pp. 13-56.

Barral L., Heinichen-Chiapella G., Simone S. (1978) - Datazione relativa del giacimento di Loreto. Atti XX Riun. Sc. Ist. It. Preist. Protost., Firenze, pp. 125-132.

Barral L. et Simone S. (1983) - Le bassin fluvio-lacustre de Venosa. Bull. Musée Anthrop. Préhist. de Monaco, 27, pp. 5-20.

Barral L. et Simone S. (1984) - Venosa-Loreto, Basilicata. in I primi abitanti d'Europa, De Luca ed., pp. 181-186.

Belli G., Belluomini G., Cassoli P.F., Cecchi S., Cucarzi M., Delitala L., Fornaciari G., Mallegni F., Piperno M., Segre A.G. et Segre-Naldini E. (1991) - Découverte d'un fémur humain acheuléen à Notarchirico (Venosa, Basilicata). L'Anthropologie (Paris), t. 95, 1, pp. 47-88.

Blanc A.C. (1953) - Venosa, gisement à industrie tayacienne et micoquienne de Loreto. IV Congr. Intern. INQUA, Livret guide pp. 63-68.

Boenzi F., La Volpe L., Rapisardi L. (1987) - Evoluzione geomorfologica del complesso vulcanico del Monte Vulture (Basilicata). Bol. Soc. Geol. It.,106, pp. 673-682.

Bonadonna F.P., Brocchini D., Laurenzi M.A., Principe C., Ferrara G. (1993) - Mt. Vulture Volcano Chronostratigraphy and Paleogeographic Implications. Symp. INQUA, Quaternary stratigraphy in volcanic areas, Roma, Abstracts, p. 13.

Bonifay M.F. (1977) - Liste préliminaire de la grande faune du gisement préhistorique de Venosa (Basilicata, Italie) : fouilles 1974-76. Bull. Musée Anthrop. Préhist. de Monaco, 21, pp. 116-125.

Caloi L. Palombo M.R. (1979a) - La fauna quaternaria di Venosa: Bovidi. Boll. Soc. Geol. It., 100, pp. 101 140.

Caloi L. Palombo M.R. (1979b) - La fauna quaternaria di Venosa: Canis sp.. Quaternaria XXI, pp. 115-128.

Caloi L. Palombo M.R. (1979c) - Megaceros solilhacus ROBERT da Terranera (Bacino di Venosa, Potenza). Quaternaria, XXI, pp. 129-138.

Cassoli P.F., Piperno M., Tagliacozzo A., Lefèvre D., Raynal J.P. (1991) - Una paleosuperficie con resti di Elefante antico e industria nel sito acheuleano di Notarchirico. Atti XXX Riun. Sc. Ist. It. Preist. Protost., Firenze, à paraître.

Chiappella G. (1964) - II Paleolitico inferiore di Venosa. Bull. Palet. It., 73, pp. 7-23.

Cortini (1975): Età K-Ar del Monte Vulture (Lucania), Riv. Ital. Geof.,2, pp. 45-46.

Daugas J.P. et Raynal J.P. (1989) - Quelques étapes du peuplement du Massif Central français dans leur contexte paléoclimatique et paléogéographique. In LAVILLE $H$. : Variations de paléomilieux et peuplement préhistorique, CNRS Ed., Cahiers du Quaternaire, 1989, n 13, 67-95.

Daugas J.P. et Raynal J.P. (1991a) - L'homme et les volcans : mésolithisation et néolithisation dans le Massif Central français. 113e Congr. nat. Soc. sav., 1988, Strasbourg, Mésolithique et Néolithisation, 127-146, 3 fig., 3 tabl. 
Daugas J.P. et Raynal J.P. (1991b) - Les chasseurs de la préhistoire face aux dangers du volcanisme. La dépèche du parc, Bull. d'information du parc naturel régional des volcans d'Auvergne,supplément scientifique, $\mathrm{N}^{\circ} 5$, juin $1991,4-5$.

De Lorenzo G. (1906) - Venosa e la regione del Vulture. Bergamo. 116p.

De Lorenzo G., D'Erasmo G. (1932) - L'Uomo palelitico e l'Elephas antiquus nell'Italia meridionale. Mem. $R$. Accad. Sc. Fis. Mat. , Napoli, s.2, 19, pp. 8-11.

D'Erasmo G. (1932) - La faune di grotta di Loretello presso Venosa. Atti R. Accad. Sc. Fis. Mat. , Napoli, s.4, 11, pp.1-22.

Durante S., Settepassi F. (1978) - Nota sulle associazioni macologiche del giacimento fluvio lacustre di Loreto, Venosa. Atti XX Riun. Sc. Ist. It. Preist. Protost., Firenze, pp.141-145.

Falguères C., Miallier D., Sanzelle S., Faïn J., Laurent M., Montret M., Pilleyre Th., Bahain J.J. (1994) Potential use of the $E^{\prime}$ center as an indicator of initial resetting in TL/ESR dating volcanic materials. Radiation Measurements, 23, pp.00-00

Guiscardi C. (1880) - Di alcuni nuovi manufatti rinvenuti a Venosa. Rend. Accad. Sc. Fis. Mat., Napoli, s.1, 19, pp.39-40.

Hashimoto T. and Habuki H. (1987) Emission properties of thermoluminescence from natural quartz. Blue and red response to absorbed dose, Nuclear Tracks and Radiation Measurements, 13 (1), pp.216-218.

Laurenzi M.A., Brocchini D., Principe C., Ferrara G. (1993) - Mt. Vulture volcano chronostratigraphy and the effectiveness of dating young phlogopites. comm. orale EUG VII, Strasbourg.

La Volpe L., Principe C. (1990) - Stratigrafia e storia eruttiva del Monte Vulture: revisione e aggiornamenti. Boll. G.N.V., 1989-2, pp.889-902.

Lefèvre D., Raynal J.P., Pilleyre T., Vernet G. (1991) - Atti XXX Riun. Sc. Ist. It. Preist. Protost., Firenze, à paraître.

Lefèvre D., Raynal J.P., Vernet G. (1993) - Sedimentary Dynamics and Tecto-Volcanism in the Venosa Basin (Basilicata, Italy). Symp. INQUA, Quaternary stratigraphy in volcanic areas, Roma, p. 43.

Miallier D., Faïn J., Montret M., Pylleyre Th., Sanzelle S. and Soumana S.(1991)Properties of the red TL peak of quartz relevant to thermoluminescence dating, Nuclear Tracks and Radiation Measurements, 18 $(1 / 2)$, pp. $89-94$

Mochi A. (1913) - Nuove osservazioni sul Paleolitico di Terranera, della Maiella e dell'Umbria. Archiv. Anthrop. Etnol., 43, Firenze.

Mochi A. (1916) - II Paleolitico di Terranera in Basilicata, secono nuove ricerche. Archiv. Anthrop. Etnol., 45, (3-4), Firenze, p. 328-337.

Neboit R. (1975): Plateaux et collines de Lucanie orientale et des Pouilles ; étude géomorphologique. Thèse, Paris, $715 \mathrm{p}$.

Nicolucci G. (1877) - Scoperte preistoriche nella basilicata e nelle Capitanata. Rend. R. Accad. Sc. Lett., Napoli, 13 p.

Picarreta G. et Ricchetti G. (1970) - I depositi del bacino fluvio-lacustre della fiumara di Venosa Mattinella e del torrente Basentello. Studio petrographico. Mem. Soc. Geol. It, 9, pp. 121-134. 
Pilleyre T. (1991) - Datation par thermoluminescence. Application à la chronologie des retombées volcaniques. Thèse de I'Université de Clermont II, DU 345, 164 p.

Pilleyre Th., Montret M., Faïn J., Miallier D. and Sanzelle S. (1992) Attempts at dating ancient volcaneos using the red TL of quartz. Quaternary Science Rewiews, Vol. 11, pp. 13-17

Pinto G., (1929) - La stazione preistorica di Venosa. Como, Ostinelli Ed., 35p.

Piperno M. (1987) - Le gisement acheuléen de Notarchirico (Venosa-Basilicata), II Congr. Intern. di Paleontologia Umana, Torino, Excursion, pp. 48-54.

Piperno M., Mallegni F., Yokoyama Y. (1990) - Découverte d'un fémur humain dans les niveaux acheuléens de Notarchrico (Venosa-Basilicata), C.R. Acad. Sc. Paris, série II, pp. 1097-1102.

Piperno M. et Segre A.G. (1982) - Pleistocene e Paleolitico inferiore di Venosa, nuove ricerche. Atti XXIII Riun. Sc. Ist. It. Preist. Protost., Firenze, pp. 589-596.

Raynal J.P. et Daugas J.P. (1984) - Volcanisme et occupation humaine dans le Massif Central français : quelques observations. Revue Archéologique du Centre de la France, t. 23, 1, 7-19.

Raynal J.P. et Daugas J.P. (1989) - Le peuplement paléolithique d'Auvergne. La Recherche , $\mathrm{n}^{\circ} 210$, vol. 20, 690-691.

Raynal J.P. et Daugas J.P. (1991) - L'homme et les volcans : occupation de l'espace régional à la fin des temps glaciaires dans le Massif Central français. in Le peuplement magdalénien. Paléogéographie physique et humaine, Actes du Colloque de Chancelade, CTHS Ed. . 11-120

Raynal J.P. et Sanzelle S. (1989) - Préhistoire en domaine volcanique : exemple de la Basse Auvergne. in Le Temps de la Préhistoire, Société préhistorique française et Archéologia Ed., tome 1, 128-129.

Rellini U. (1915) - Sulle stazioni quaternarie di tipo Chelléen dell'Agro Venosino. Mem. Accad. Lincei, 312 , 15, s.5, pp. 181-210.

Rellini U. (1932a) - Sulla scoperta di uno strato pre-amigdaliano a Loretello di Venosa e sugli indizi probabili di un'età protolitica in Italia. Bull. Paletn. It., 50-51, (1930-31), p. I-II.

Rellini U. (1932b) - La fauna dello strato pre-amigdaliano di Loretello di Venosa. Bull. Paletn. It., 52, (1930-31), p. 1-4.

Sala (1991) - Primi dati sui microvertebrati di Notarchirico, Preistoria Alpina, sous presse.

Segre A.G. (1978) - II Pleistocene e il Paleolitico della Basilicata; Atti XX Riun. Sc. Ist. It. Preist. Protost., Firenze, pp. 15-39.

Segre A.G., Bidittu I., et Piperno M. (1982) - II Paleolitico inferiore nel Lazio, nella Basilicata e in Sicilia. Atti XXIII Riun. Sc. Ist. It. Preist. Protost., Firenze, pp. 177-206.

Segre A.G. et Piperno M. (1984) - Venosa-Notarchirico, Basilicata. in Primi abitanti d'Europa, De Luca ed.,pp. 186-188.

Topa D. (1932) - La collezione paleolitica Briscese e la Grotta di Loreto presso venosa in Basilicata. Palmi, 321 p. 